\title{
Protein C (OprC) of the outer membrane of Pseudomonas aeruginosa is a copper-regulated channel protein
}

\author{
Hiroshi Yoneyama and Taiji Nakae \\ Author for correspondence: Hiroshi Yonevama. Tel: +81463931121 . Fax: +81463962892. \\ e-mail: yoneyama@is.icc.u-tokai.ac.jp
}

Department of Molecular Life Science, Tokai University, School of Medicine, Isehara 259-11, Japan

\begin{abstract}
Protein C (OprC) of the outer membrane of Pseudomonas aeruginosa forms small channels, as assayed by the liposome swelling method. We report here that OprC functions as a channel-forming and copper-binding protein. OprC purified to homogeneity formed a channel in planar lipid bilayers with an ion conductance of about $200 \mathrm{pS}$ in $1 \mathrm{M} \mathrm{NaCl}$. Cloning and sequencing of the gene encoding OprC revealed that it specified a polypeptide comprising 723 and 668 amino acid residues for the precursor and mature polypeptides $\left(M_{r} 73372\right)$, respectively. The amino acid sequence of OprC showed the highest degree of similarity with that of NosA of Pseudomonas stutzeri (65\% sequence identity) which conveys $\mathrm{Cu}^{2+}$ to intracellular acceptor(s). OprC showed high copperbinding activity $\left(K_{\mathrm{d}}=2.6 \mu \mathrm{M}\right)$ in aqueous solution containing surfactant. The expression of OprC appeared to be repressed by exogenous $\mathrm{Cu}^{2+}$ and derepressed by anaerobiosis in the presence of nitrate. These results suggest that OprC might be involved in copper utilization.
\end{abstract}

Keywords: Pseudomonas aeruginosa, porin, protein C (OprC), copper-binding protein, outer membrane

\section{INTRODUCTION}

The outer membrane of Pseudomonas aeruginosa forms a tight barrier against the penetration of large hydrophilic solutes. This barrier function is due largely to the predominance of small diffusion pores (Yoneyama et al., 1986; Yoneyama \& Nakae, 1986). Using the liposome swelling assay, we identified outer-membrane proteins (OMPs) OprC, OprD2 and OprE1 to be the proteins having a porin function that allowed the diffusion of small hydrophilic solutes, such as saccharides, with $M_{\mathrm{r}}$ below 350-400 (Yoshihara \& Nakae, 1989). Additionally, studies using planar lipid bilayers have shown that OprD2 and OprE1 form smaller ion conductive channels than those of the Escherichia coli porins (Ishii \& Nakae, 1993; Obara \& Nakae, 1992).

Biochemical analysis shows that OprD2 is equipped with a gate- and a channel-forming domain, suggesting that it somehow regulates channel activity by this means (Yoshihara \& Nakae, 1992). Flickering of the OprD2-

Abbreviations: $\mathrm{C}_{12} \mathrm{E}_{\mathrm{g}}$, octaethylene glycol dodecylether; diPhy-PC, $\mathrm{L}-\alpha-$ diphytanoylphosphatidyl choline; OMP, outer-membrane protein.

The DDBJ accession number for the nucleotide sequence reported in this paper is D28119. channel was also demonstrated using planar lipid bilayers in the presence of lipopolysaccharide (Ishii \& Nakae, 1993), consistent with the finding of the gate domain. Earlier studies showed that OprD2 serves as a selective diffusion pore for a small $\beta$-lactam antibiotic, imipenem (Büscher et al., 1987; Quinn et al., 1986; Satake et al., 1990; Trias \& Nikaido, 1990; Yoneyama \& Nakae, 1991). The primary structures of OprD2 and OprE1 have been deduced from the nucleotide sequences of $o p r D$ and oprE, respectively, and showed that the proteins have a high degree of similarity to each other (Yoneyama et al., 1992; Yamano et al., 1993).

In contrast, the physiological function of OprC remains to be elucidated. For a better understanding of the physiological function of $\mathrm{OprC}$, we investigated the channel activity of $\mathrm{OprC}$ in planar lipid bilayers and cloned the $\operatorname{oprC}$ gene. Here, we report that $\mathrm{OprC}$ is the channelforming and copper-binding protein. We also discuss the bifunctionality of OprC in terms of copper utilization.

\section{METHODS}

Bacterial strain, plasmid and growth conditions. $P$. aeruginosa PAO1 was grown aerobically in L-broth containing $10 \mathrm{~g}$ Tryptone (Difco), $5 \mathrm{~g}$ Yeast Extract (Difco), and $5 \mathrm{~g} \mathrm{NaCl} \mathrm{l}^{-1}$ $(\mathrm{pH} 7 \cdot 2)$ at $37^{\circ} \mathrm{C}$ overnight or anaerobically in L-broth con- 
taining $40 \mathrm{mM} \mathrm{KNO}_{3}$. DNA was cloned in E. coli XL1-Blue (Stratagene) and JM109 (Toyobo). A P. aeruginosa chromosomal DNA library was constructed and the isolated DNA fragment was cloned using the phagemid vector pBluescript II SK(+) (Stratagene).

Formation of planar lipid bilayers and conductivity measurements. We formed planar lipid bilayers by the procedure described previously (Ishii \& Nakae, 1993; Obara \& Nakae, 1992; Benz et al., 1978). A Teflon chamber, separating two aqueous phases by a septum having an orifice of $0.5-1 \mathrm{~mm}$ in diameter, was used. The orifice edge was precoated with about $0.3 \mu \mathrm{l}$ of a $2 \mathrm{mg} \mathrm{ml}^{-1}$ solution of $\mathrm{L}$ - $\alpha$-diphytanoylphosphatidyl choline (diPhy-PC) dissolved in hexane. Two microlitres of a $10 \mathrm{mg} \mathrm{ml}^{-1}$ solution of diPhy-PC, dissolved in $\mathrm{n}$-decane on a brass wire sleeved with Teflon tubing, was applied to the orifice of the septum immersed in a salt solution containing $1 \mathrm{M} \mathrm{NaCl}$ and $2.5 \mathrm{mM}$ HEPES ( $\mathrm{pH} 7.0$ ) in the chamber. Electrical resistance of the membrane appeared to be consistently higher than $400 \mathrm{G} \Omega \mathrm{cm}^{-2}$. The protein to be tested was added to the cis compartment in which a $10 \mathrm{G} \Omega$ probe was immersed. The conductivity was recorded through $\mathrm{Ag} / \mathrm{AgCl}_{2}$ electrodes connected to a patch-clamp amplifier CEZ-2200 (Nihon-Koden) in voltage-clamping mode. A membrane potential was applied through a stimulator (SEN-3301, Nihon-Koden). Signals were recorded by a digital recording oscilloscope 2232 (Tektronix) and a pen recorder LR4201 (Yokogawa).

Cloning of oprC. We cloned and sequenced oprC according to standard procedures (Sambrook et al., 1989). The DNA library of $P$. aeruginosa PAO1 was constructed by ligating Sau3AIdigested DNA fragments $(4-10 \mathrm{~kb})$ with BamHI-digested pBluescript II SK(+) using E. coli XL1-Blue as the host strain (Yoneyama \& Nakae, 1991). Transformants were screened using the rabbit anti-OprC antibody (Yoneyama \& Nakae, 1991; Yoshihara et al., 1991).

Immunological screening and Western blots. Rabbit anti$\mathrm{OprC}$ antiserum was prepared as described previously (Yoshihara et al., 1991). The antibody was further purified by means of a Sepharose 4B column with immobilized OprC. Immunological screening and Western blotting were carried out as described previously (Yoneyama \& Nakae, 1991; Yoshihara et al., 1991).

DNA sequencing and analysis. Nested sets of deletion plasmids were prepared by digesting plasmid DNA with exonuclease III and mung bean nuclease (Yoneyama et al., 1992; Sambrook et al., 1989). Both coding and non-coding strands were sequenced by the dideoxy chain-termination method (Sanger et al., 1977) using BcaBEST (Takara) and Sequenase Version 2.0 (USB). The derived sequence was analysed using TBLASTN software (Altschul et al., 1990), from the NCBI BLAST server, and also TFASTA software (Lipman \& Pearson, 1985; Pearson \& Lipman, 1988 ) implemented on a FLAT DB e-mail network server.

Restriction map of cloned gene. Restriction maps of DNA from the anti-OprC antibody-positive clones were constructed by digesting recombinant plasmids with one or a combination of several restriction enzymes (Yoneyama et al., 1992).

Purification of OprC and determination of the $\mathrm{N}$-terminal amino acid sequence. Mature $\mathrm{OprC}$ was purified as described previously (Yoshihara \& Nakae, 1989) with minor modifications. The fraction containing OprC eluted from a DEAEHPLC column (TSKgel DEAE-5PW, $7.5 \times 0.75 \mathrm{~cm}$ i.d.) was mixed with a solution containing $2 \mathrm{M}\left(\mathrm{NH}_{4}\right)_{2} \mathrm{SO}_{4}$ and $0.01 \%$ octaethylene glycol dodecylether $\left(\mathrm{C}_{12} \mathrm{E}_{8}\right)$. The mixture was applied to a hydrophobic HPLC column (TSKgel Ether-5PW, $7.5 \times 0.75 \mathrm{~cm}$ i.d.) equilibrated with $100 \mathrm{mM}$ Tris $/ \mathrm{HCl}(\mathrm{pH}$
7.5), $2 \mathrm{M}\left(\mathrm{NH}_{4}\right)_{2} \mathrm{SO}_{4}, 0.01 \% \mathrm{C}_{12} \mathrm{E}_{8}$ and $20 \%(\mathrm{v} / \mathrm{v})$ glycerol, and the column was eluted with a linear gradient of $\left(\mathrm{NH}_{4}\right)_{2} \mathrm{SO}_{4}$ $(2-0 \mathrm{M})$ in $100 \mathrm{mM}$ Tris/ $\mathrm{HCl}(\mathrm{pH} 7.5)$ containing $0.04 \% \mathrm{C}_{12} \mathrm{E}_{8}$ and $20 \%$ glycerol. After dialysis against a large excess of $10 \mathrm{mM}$ Tris/ $\mathrm{HCl}$ ( $\mathrm{pH} 8$ ) containing $1 \mathrm{mM} \mathrm{C}_{12} \mathrm{E}_{8}$, the OprC preparation was resolved by the SDS-PAGE $(10 \%, \mathrm{w} / \mathrm{v}$, polyacrylamide) and the protein band was blotted on a PVDF membrane at $1 \mathrm{~mA}$ $\mathrm{cm}^{-2}$ for $45 \mathrm{~min}$ (Hirano \& Watanabe, 1990). The OprC band was excised and subjected to Edman degradation in a gas-phase sequenater (Model 477A, Applied Biosystems).

Determination of the copper-binding activity of OprC. A Teflon cell comprising two chambers separated by a dialysis membrane (Spectrum Medical Industries) was used for equilibrium dialysis. One chamber was filled with purified OprC $(2 \cdot 9 \mu \mathrm{M})$ in a solution containing $5 \mathrm{mM} \mathrm{C}_{12} \mathrm{E}_{8}, 10 \mathrm{mM} \mathrm{NaCl}$ and $10 \mathrm{mM}$ Tris/ $\mathrm{HCl}(\mathrm{pH} 7 \cdot 5)$. Another chamber contained an appropriate concentration of $\mathrm{CuSO}_{4}$ dissolved in the same solution. After dialysis by rotation at 16 r.p.m. using a 5-Cell Equilibrium Dialyzer (Spectrum) at $20^{\circ} \mathrm{C}$ for $2 \mathrm{~h}$, the $\mathrm{Cu}^{2+}$ concentration in both compartments was determined as described below.

Other techniques. $\mathrm{Cu}^{2+}$ was quantified according to the method of Hirayama (1976) with some modifications. A sample $(50 \mu \mathrm{l})$ was placed in a microtube containing $20 \mu \mathrm{l} 1 \mathrm{M}$ ammonium buffer $(\mathrm{pH} 9 \cdot 3)$ and $350 \mu \mathrm{l}$ water. After the addition of $82.2 \mu \mathrm{l}$ $0.05 \%$ o-aminophenol (Wako) containing $0.005 \mathrm{M}$ sulphuric acid, the mixture was incubated at $23^{\circ} \mathrm{C}$ for $30 \mathrm{~min}$, then chilled on ice for $2 \mathrm{~min}$. The oxidized phenoxazine derivative was extracted with $500 \mu \mathrm{l}$ chloroform and the chloroform phase was dried with about $100 \mathrm{mg} \mathrm{Na} \mathrm{SO}_{4}$. Developed colour was determined spectrophotometrically (Shimadzu, Model UV2200 ) at $414 \mathrm{~nm}$ (path length $1 \mathrm{~cm}$ ). Protein was quantified by the method of Lowry. SDS-PAGE was carried out according to the method of Laemmli (1970).

\section{RESULTS}

\section{Channel activity of OprC}

We have previously shown, by the liposome swelling method, that OprC functions as a porin (Yoshihara \& Nakae, 1989). To test channel activity using different methodology, we measured the ion conductivity of the protein reconstituted in planar lipid bilayers. When about $1.6 \times 10^{-15} \mathrm{~mol}$ of highly purified $\mathrm{OprC}$ was added into the cis compartment of the chamber filled with $1 \mathrm{M}$ $\mathrm{NaCl} / 2.5 \mathrm{mM}$ HEPES buffer ( $\mathrm{pH} 7.0$ ), stepwise conductance increments were observed (Fig. 1a). A histogram of the conductance showed two discrete peaks at about 200 and $450 \mathrm{pS}$ (Fig. 1b). The channels showed occasional closing with a conductance of about $200 \mathrm{pS}$ (Fig. 1c). These results suggest that the mean single channel conductance of $\mathrm{OprC}$ is about $200 \mathrm{pS}$ in $1 \mathrm{M} \mathrm{NaCl}$ and that the large conductance values of about $400 \mathrm{pS}$ might be the result of simultaneous insertion of two channel units or because the protein exists in two different conformational states.

\section{Cloning, mapping and nucleotide sequencing of the oprC gene}

We isolated three anti-OprC antibody-reactive clones by screening a chromosomal DNA library of $P$. aeruginosa PAO1 constructed in E. coli XL1-Blue. Restriction maps 
(a)

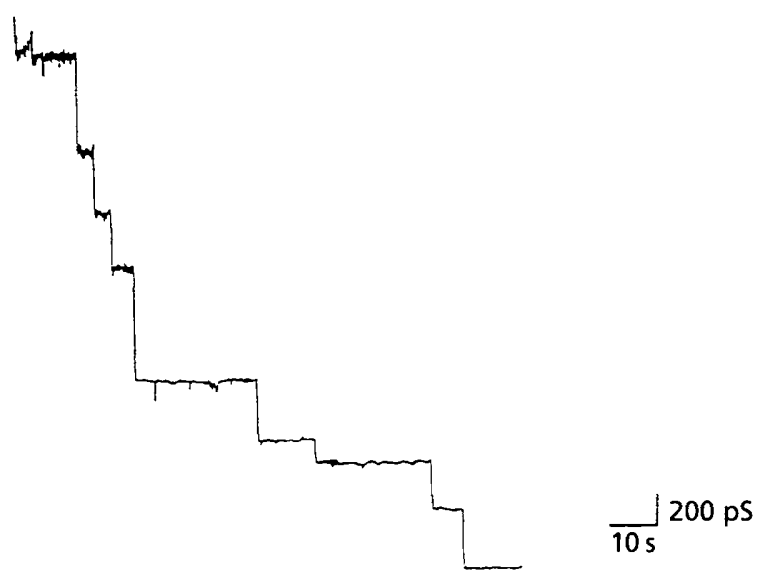

(b)

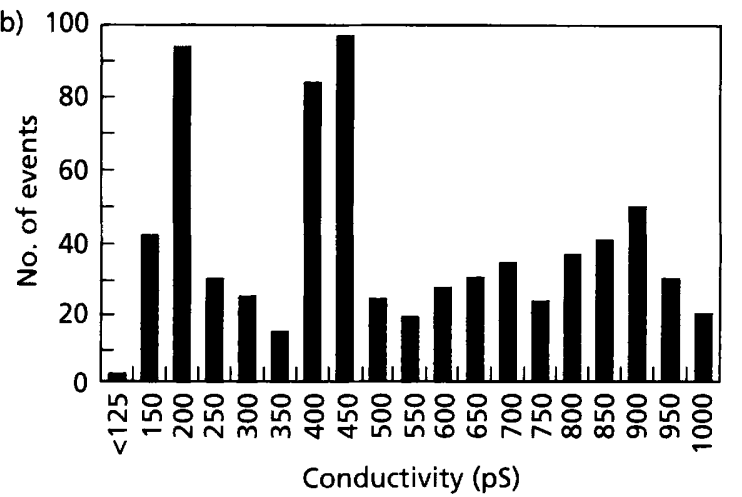

(c)

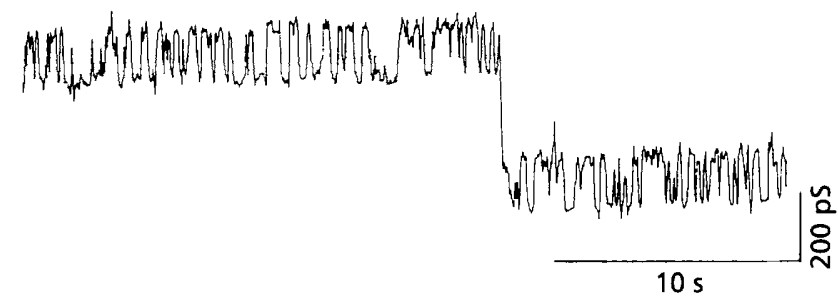

Fig. 1. Channel conductivity of OprC in a diPhy-PC membrane. (a) Typical conductivity increments in the presence of OprC. Purified OprC (about $1.6 \times 10^{-15} \mu \mathrm{mol}$ ) in $5 \mathrm{mM} \mathrm{C} \mathrm{C}_{12} \mathrm{E}_{8}, 1 \mathrm{mM}$ EDTA, $10 \mathrm{mM}$ Tris/ $\mathrm{HCl}(\mathrm{pH} 8)$ was added to the ciscompartment. The final $\mathrm{C}_{12} \mathrm{E}_{8}$ concentration was less than $2 \mu \mathrm{M}$ and this concentration of $\mathrm{C}_{12} \mathrm{E}_{8}$ showed no detectable influence on the conductivity. The applied potential was $+50 \mathrm{mV}$. (b) Distribution of channel conductance. The histogram represents 717 independent channel events. (c) An example of flickering of OprC channel.

of the three clones indicated chromosomal DNA inserts of about $5 \cdot 7,6.5$ and $8 \mathrm{~kb}$, respectively. However, restriction sites over a $4.5 \mathrm{~kb}$ region of each of the DNA fragments were common to all three (data not shown). Additionally, a protein band corresponding to $\mathrm{OprC}$ on an electrophoretogram of total cellular extracts from each transformant was reactive with anti-OprC antibody (data not shown). To localize the region containing the oprC (a)

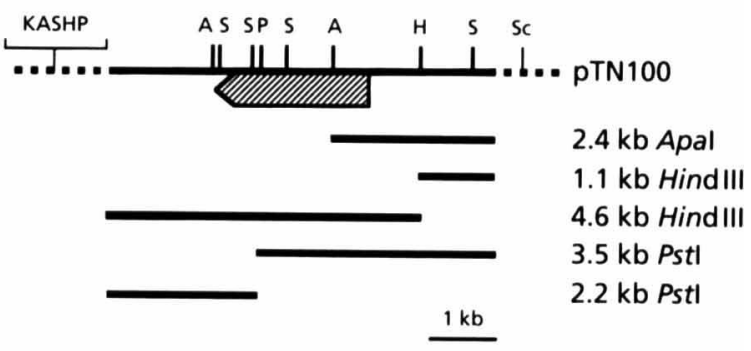

(b)
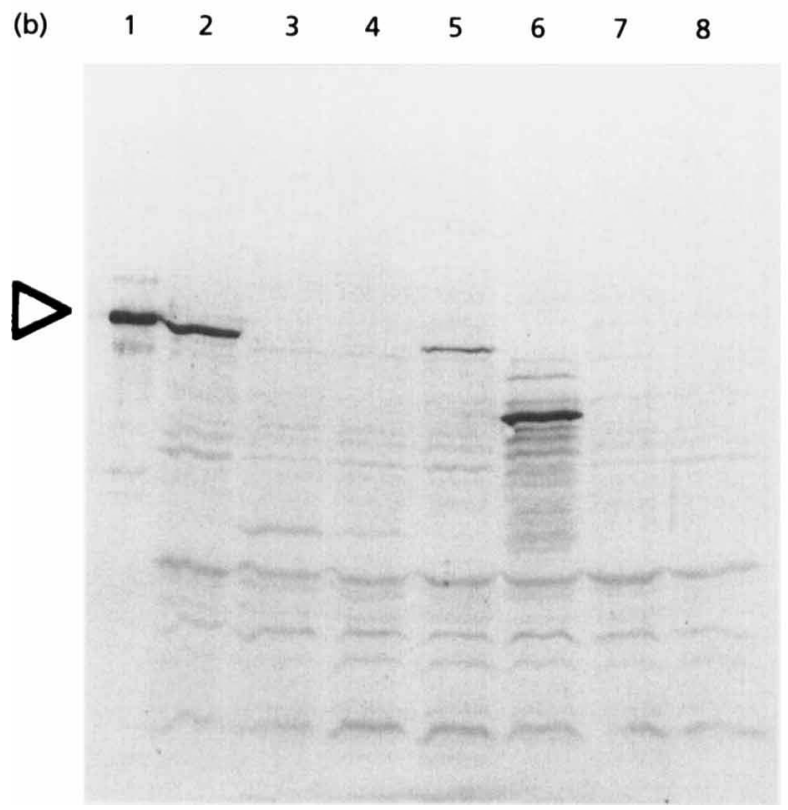

Fig. 2. Restriction fragments and Western blot analysis of the products from the genes in the fragments. (a) Restriction fragments derived from pTN100 were subcloned. The hatched box indicates the location of the structural gene for OprC. A, Apal; H, Hindlll; K, Kpnl; P, Pstl; S, Sall; Sc, Sacl. (b) Western blots of whole cell lysates from $E$. coli XL1-Blue harbouring DNA inserts subcloned into pBluescript || SK $(+)$. Lanes: 1, purified outer membrane of $P$. aeruginosa PAO1; $2-7, E$. coli XL1-Blue harbouring pTN100 (2), the $2.4 \mathrm{~kb}$ Apal fragment (3), the $1.1 \mathrm{~kb}$ Hind III fragment (4), the $4.6 \mathrm{~kb}$ Hind III fragment (5), the $3.5 \mathrm{~kb}$ Pstl fragment (6) and the $2.2 \mathrm{~kb}$ Pstl fragment (7); $8, E$. coli XL1Blue. The amounts of protein applied were $2 \mu \mathrm{g}$ for lane 1 and $30 \mu \mathrm{g}$ for lanes 2-8. The arrowhead indicates the location of mature OprC.

gene, we subcloned ApaI, HindIII and Pst I fragments into the phagemid vector pBluescript II SK(+) (Fig. 2a). A transformant harbouring a plasmid with an insert of $5.7 \mathrm{~kb}$ (pTN100) or a plasmid containing a $4.6 \mathrm{~kb}$ HindIII fragment produced a single band corresponding to authentic $\mathrm{OprC}$ as determined by immunoblotting analysis (Fig. 2b, lanes 2 and 5). Transformants harbouring the plasmid containing other fragments showed undetectable or truncated proteins. Since the $M_{\mathrm{r}}$ of $\mathrm{OprC}$ is about 70000 (Yoshihara \& Nakae, 1989), the size of $\operatorname{opr} C$ was expected to be around $2 \cdot 1 \mathrm{~kb}$. Therefore, we concluded that the location of the $5^{\prime}$ end of $\operatorname{opr} C$ would be close to 


\begin{tabular}{|c|c|c|c|c|c|c|}
\hline $\begin{array}{l}\text { Oprc } \\
\text { NosA }\end{array}$ & $\begin{array}{l}---390 \\
\text { AESVDIISEMA }\end{array}$ & 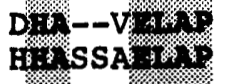 & 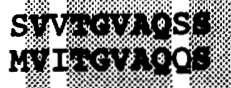 & 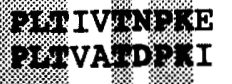 & 8.098919 .96 & $\begin{array}{l}43 \\
50\end{array}$ \\
\hline $\begin{array}{l}\text { OprC } \\
\text { NosA }\end{array}$ & 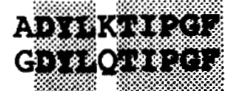 & 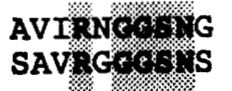 & 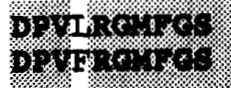 & $\begin{array}{l}\text { 3NI THEGM } \\
\text { 39KLA }\end{array}$ & 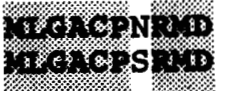 & $\begin{array}{r}93 \\
100\end{array}$ \\
\hline $\begin{array}{l}\text { OprC } \\
\text { NosA }\end{array}$ & 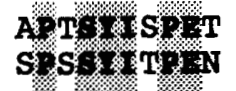 & WOK & 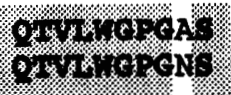 & 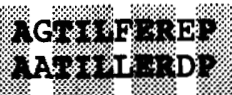 & 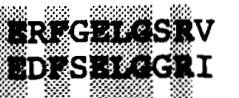 & $\begin{array}{l}143 \\
150\end{array}$ \\
\hline $\begin{array}{l}\text { Oprc } \\
\text { NosA }\end{array}$ & 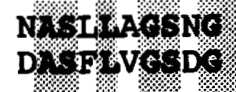 & 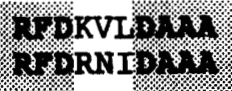 & $\begin{array}{l}\text { CNRLCILIFTT } \\
\text { GGEOSIILL }\end{array}$ & 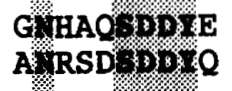 & WAENTMPIS & $\begin{array}{l}193 \\
200\end{array}$ \\
\hline $\begin{array}{l}\text { OprC } \\
\text { NosA }\end{array}$ & $\begin{array}{l}\text { MYNGPVAV } \\
\text { MOSTHLV }\end{array}$ & 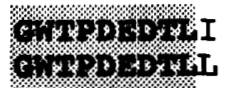 & 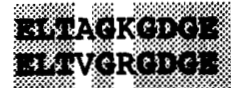 & 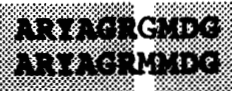 & 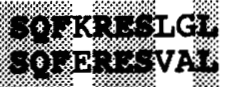 & $\begin{array}{l}243 \\
250\end{array}$ \\
\hline $\begin{array}{l}\text { OprC } \\
\text { NosA }\end{array}$ & $\begin{array}{l}\text { PVISTSDV } \\
\text { POE TILEN }\end{array}$ & MEIVIOY) & 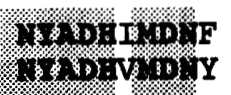 & 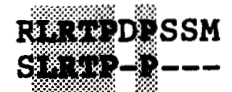 & PMP & $\begin{array}{l}293 \\
295\end{array}$ \\
\hline $\begin{array}{l}\text { OprC } \\
\text { NosA }\end{array}$ & mingering & 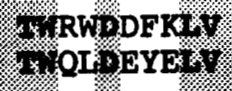 & 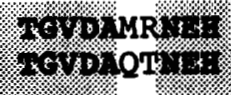 & $\begin{array}{l}\text { LAMSSKYDMM } \\
\text { SR:M G- }\end{array}$ & $\begin{array}{l}\text { TPFYYTDADQF } \\
\text { VPYKSK- }\end{array}$ & $\begin{array}{l}343 \\
336\end{array}$ \\
\hline $\begin{array}{l}\text { Oprc } \\
\text { NosA }\end{array}$ & 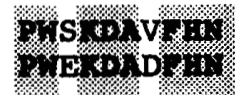 & 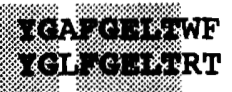 & $\begin{array}{l}\text { AAERD IIFe? } \\
\text { LNDDS VYI }\end{array}$ & 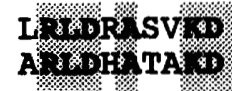 & 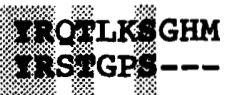 & $\begin{array}{l}393 \\
383\end{array}$ \\
\hline $\begin{array}{l}\text { Oprc } \\
\text { NosA }\end{array}$ & $\begin{array}{l}\text { GHAMANPTRN } \\
-\end{array}$ & 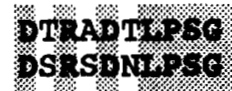 & 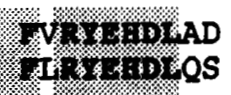 & $\begin{array}{l}\text { SPTLLACS } \\
\text { LAAAVES }\end{array}$ & 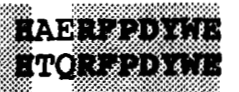 & $\begin{array}{l}443 \\
425\end{array}$ \\
\hline $\begin{array}{l}\text { OprC } \\
\text { NosA }\end{array}$ & H. & 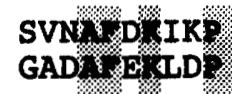 & 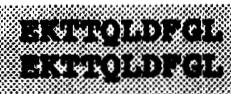 & $\begin{array}{l}\text { YNGDKSOM } \\
\text { OS SKGP }\end{array}$ & 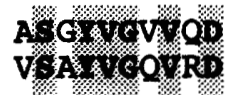 & $\begin{array}{l}493 \\
469\end{array}$ \\
\hline $\begin{array}{l}\text { Oprc } \\
\text { NosA }\end{array}$ & 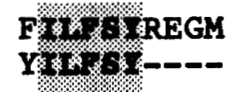 & $\begin{array}{l}\text { MGS TQAT } \\
\text {-SPSKYSE I }\end{array}$ & 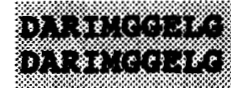 & 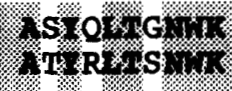 & 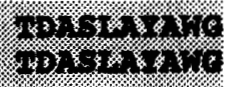 & $\begin{array}{l}543 \\
514\end{array}$ \\
\hline $\begin{array}{l}\text { OprC } \\
\text { NosA }\end{array}$ & 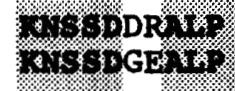 & OIPURARFI & 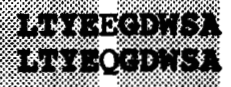 & $\begin{array}{l}\mathrm{GS} \\
\mathrm{AG}\end{array}$ & IIVRDQ $\%$ & $\begin{array}{l}593 \\
564\end{array}$ \\
\hline $\begin{array}{l}\text { OprC } \\
\text { NosA }\end{array}$ & 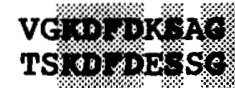 & 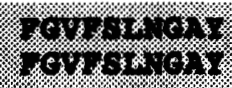 & $\begin{array}{l}\text { WTR V } \\
\text { HNOFE }\end{array}$ & 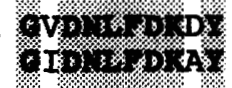 & 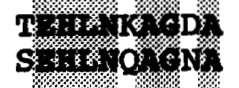 & $\begin{array}{l}643 \\
614\end{array}$ \\
\hline $\begin{array}{l}\text { OprC } \\
\text { NosA }\end{array}$ & $\begin{array}{l}\text { GFEF YNUTV } \\
\text { EICISIDLRI }\end{array}$ & 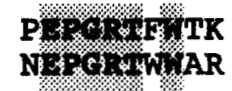 & Jopsis & & & $\begin{array}{l}668 \\
639\end{array}$ \\
\hline
\end{tabular}

Fig. 3. Alignment of the amino acid sequence of the mature polypeptides of OprC and NosA. The alignment was carried out using the software package GENEwORKs (IntelliGenetics). Gaps were introduced to optimize the alignment. Identical amino acid residues are shaded.

the ApaI site located between the HindIII and PstI sites, and that the $3^{\prime}$ end would be near another $A$ paI site (Fig. 2a).
The coding strand of the $4.6 \mathrm{~kb}$ HindIII fragment and the anticoding strand of the $2 \cdot 2 \mathrm{~kb}$ and $3.5 \mathrm{~kb}$ Pst I fragments of pTN100 were sequenced by the dideoxy chain- 
termination method. The sequence contains an ORF having 2169 bp encoding 723 amino acid residues. There were three putative RNA polymerase binding sites: a $\sigma^{30}$ recognition site (Deretic et al., 1989) at nucleotides - 311 to -287 , an anr site (for anaerobic regulation of arginine deiminase and nitrate reduction; Galimand et al., 1991) at -124 to -111 , and $\sigma^{54}$ sites (Deretic et al., 1989) at -90 to $-77,-48$ to -35 and -44 to -31 . A ribosome binding site (GGAaGT) (Toschka et al., 1988) was located at -16 to -11 from the adenine of the initiation codon. A self-complementary sequence without a run of Ts appeared at the end of the sequence, suggesting that this site may act as a $\rho$-dependent transcription terminator (Lundrigan \& Kadner, 1986; Küpper et al., 1978; Rosenberg \& Court, 1979).

\section{Amino acid sequence of OprC}

The ORF encoded a protein of 723 amino acid residues with a calculated $M_{\mathrm{r}}$ of 79215 . To confirm whether the isolated gene encoded authentic OprC, we compared the $\mathrm{N}$-terminal amino acid sequence of purified OprC with that deduced from the nucleotide sequence of $\operatorname{opr} C$. The sequence of the OprC protein appeared to be $\mathrm{H}-\mathrm{S}-\mathrm{Q}-\mathrm{H}-$ Q-D-X-A-V-E, which was identical to a stretch of amino acids encoded by the ORF (amino acid residues 56-65). Thus, we concluded that the cloned gene encodes OprC. The second in-frame initiation codon with an appropriate 16S rRNA binding site (AtGGA) was found at the fourth amino acid residue. It is not clear at present whether OprC translation starts from either one of these initiation codons or from both sites. However, an extraordinarily long leader sequence is known to exist for the OMP of $P$. putida, the ferric-pseudobactin receptor PupA (Bitter et al., 1991). Mature OprC consists of 668 amino acid residues and the $M_{\mathrm{r}}$ appears to be 73372 . The content of hydrophobic and polar amino acids of $\mathrm{OprC}$ was similar to that of other OMPs. Moreover, the C-terminal residue of $\mathrm{OprC}$ was Phe. These are all common features of channel-forming OMPs in bacteria (Yoneyama et al., 1992; Yamano et al., 1993; Lundrigan \& Kadner, 1986; Dean \& Poole, 1993; Heller \& Kadner, 1985; Lee et al., 1991; Nau \& Konisky, 1989; Sauer et al., 1990).

\section{Sequence similarity search}

A computer-aided similarity search of the amino acid sequence of mature OprC was performed. The amino acid sequence of OprC showed the highest degree of similarity with NosA of $P$. stutzeri, with $65 \%$ sequence identity (Fig. 3). To assess the statistical similarity/dissimilarity between OprC and Nos A, we used the shuffling program, RDF2, of Lipman \& Pearson (1985) with 100 random shuffles. The degree of similarity between $\mathrm{OprC}$ and Nos A appeared to be 113.5 of the optimized $Z$ value (in terms of SD above the mean). Other proteins found to be similar to OprC were PfeA, FepA, Cir, FhuE and BtuB with optimized $Z$ values $13 \cdot 3,7 \cdot 4,6 \cdot 2,10 \cdot 2$ and $6 \cdot 3$, respectively. A $Z$ value greater than 10 indicates significant relatedness and that of $6-10$ indicates a probable relationship. Therefore, the similarity of OprC with these (a)

\begin{tabular}{lcc} 
OprC & DKLTVIKGPQTVLWGPGA & 122 \\
NOSA & DALTVIKGPQTVLWGPGN & 129 \\
PfeA & ERIEVIRGPAAARYGNGA & 139 \\
FepA & ERIEVLRGPARARYGNGA & 137 \\
Cir & ERIEVVRGPMSSLYGSDA & 123 \\
FhuE & ERVEVVRGATGLMTGTGN & 137 \\
BtuB & QRVEYIRGPRSAVYGSDA & 122 \\
& \multicolumn{2}{c}{ * }
\end{tabular}

(b)

OprC

NosA

PfeA

FepA

Cir

FhuE

BtuB

\begin{tabular}{|c|c|}
\hline SAGVDNLFDK & 631 \\
\hline LSTGIDNLFDK & 602 \\
\hline LGAGVDNLFDK & 684 \\
\hline LTGGVDNLFDK & 680 \\
\hline LRAGVLNLGDK & 614 \\
\hline LQGNVNNLFDK & 667 \\
\hline VRGKIANLFDK & 571 \\
\hline
\end{tabular}

Fig. 4. Similarity of sequences of OprC and the TonBdependent OMPs. The number of amino acid residues at the end of the similar region of the mature protein is indicated on the right. (a) A well-conserved sequence in the central region; (b) a conserved sequence in the C-terminal region. Multiple alignment was carried out using the molecular evolutionary analysis system ODEN, programmed by $Y$. Ina (National Institute of Genetics, Japan), loaded on a DDBJ computer.

proteins appeared to be statistically significant. The proteins found to be similar to OprC are the TonBdependent OMPs (Fig. 4). Multiple alignment showed that two glycine residues are conserved in the central region of OprC (Fig. $4 \mathrm{a}$ ) and 4 amino acid residues, N-L$X-D-K$ (where $X$ is $F$ or $G$ ), are conserved in the $C$ terminal region (Fig. 4b). The latter 4 amino acid residues were assumed to be the site involved in substrate binding (Lee et al., 1991).

\section{Regulation of OprC expression}

Since the amino acid sequence of OprC deduced from the nucleotide sequence showed a high degree of similarity with NosA of $P$. stutzeri, expression of which is regulated by $\mathrm{Cu}^{2+}$, we tested the effect of $\mathrm{Cu}^{2+}$ on OprC expression. When the $\mathrm{Cu}^{2+}$ concentration in the medium was increased stepwise from $0 \cdot 1$ to $100 \mu \mathrm{M}$, synthesis of OprC became clear at 0 and $0 \cdot 1 \mu \mathrm{M}$, but was barely detectable above $1 \mu \mathrm{M}$ (Fig. 5a). This result contrasts with the expression of NosA in $P$. stutzeri where $11 \mu \mathrm{M} \mathrm{Cu}^{2+}$ completely repressed its synthesis (Lee et al., 1989). To confirm that the faint band that appeared was OprC and not another protein of similar size, we probed the protein band by Western blotting (Fig. 5b). The result clearly showed the presence of OprC. The effect of other divalent cations such as $\mathrm{Pb}^{2+}, \mathrm{Mn}^{2+}, \mathrm{Ni}^{2+}, \mathrm{Cd}^{2+}, \mathrm{Co}^{2+}, \mathrm{Fe}^{2+}$ and $\mathrm{Zn}^{2+}$ at $38-90 \mu \mathrm{M}$ on OprC expression was undetectable (data not shown). Thus the effect of $\mathrm{Cu}^{2+}$ on the regulation of OprC synthesis seemed specific. Since oprC has a 

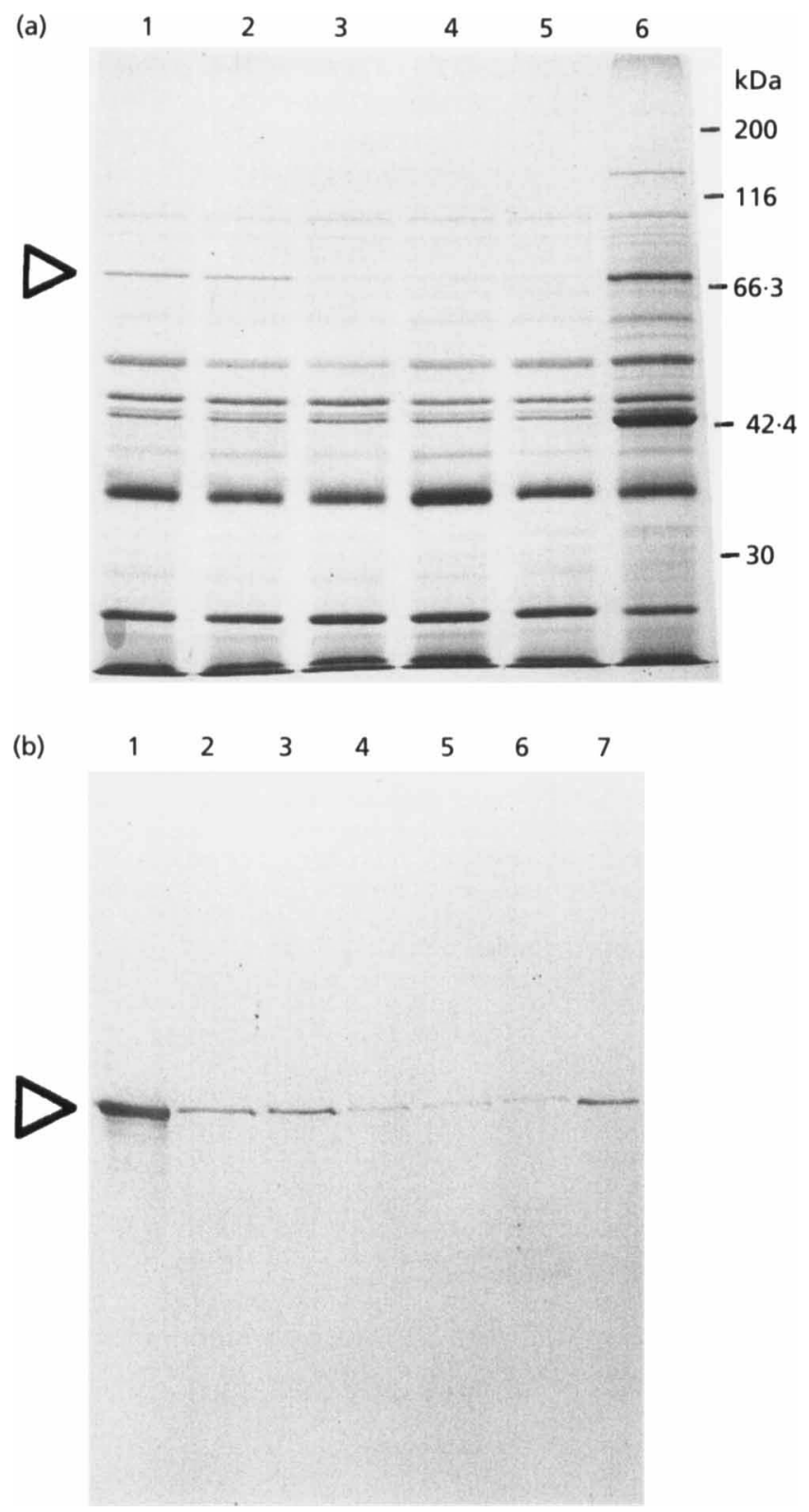

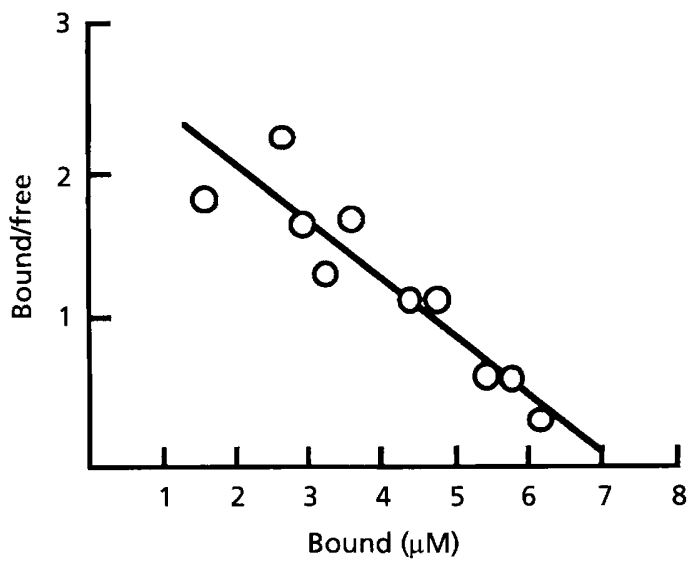

Fig. 6. Binding of $\mathrm{Cu}^{2+}$ to OprC. Data were from three independent experiments. An experiment in the presence of $100 \mathrm{mM}$ of $\mathrm{NaCl}$ showed almost the same result.

shows that $\mathrm{OprC}$ was overexpressed under anaerobic culture conditions, confirming a report by Yamano et al. (1993).

\section{Binding of $\mathrm{Cu}^{2+}$ by OprC}

Since OprC expression was regulated by $\mathrm{Cu}^{2+}$ (Fig. 5) and OprC has a high degree of similarity with NosA, the copper-binding protein, it was conceivable that OprC binds with $\mathrm{Cu}^{2+}$. We therefore determined the binding affinity of $\mathrm{Cu}^{2+}$ to highly purified $\mathrm{OprC}$ by the equilibrium dialysis method. The results depicted in Fig. 6 clearly show that $\mathrm{OprC}$ bound $\mathrm{Cu}^{2+}$. Scatchard plots showed a $K_{\mathrm{d}}$ value of $2 \cdot 6 \mu \mathrm{M}$ and two to three ligand binding sites per molecule. Since the production of OprC was derepressed under anaerobiosis in the presence of nitrate and its amino acid sequence showed a high degree of similarity with NosA, it is highly likely that OprC is also involved in copper utilization by $P$. aeruginosa.

\section{DISCUSSION}

Fig. 5. Effect of $\mathrm{CuSO}_{4}$ and $\mathrm{KNO}_{3}$ on the OMP profile of $P$ aeruginosa PAO1. Cells were grown in the presence or absence of $\mathrm{Cu}^{2+}$ and the outer membranes were purified as described in Methods. (a) Purified outer membrane $(20 \mu \mathrm{g}$ protein) was resolved by SDS-PAGE $\left(10 \%\right.$ gel). Lanes: 1 , without $\mathrm{Cu}^{2+} ; 2$, $0.1 \mu \mathrm{M} \mathrm{CuSO}{ }_{4} ; 3,1 \mu \mathrm{M}$ CuSO $_{4} ; 4,10 \mu \mathrm{M}$ CuSO $_{4} ; 5,100 \mu \mathrm{M}$ $\mathrm{CuSO}_{4} ; 6,40 \mathrm{mM} \mathrm{KNO}_{3}$. The molecular mass markers were myosin (200 kDa), $\beta$-galactosidase (116 kDa), albumin $(66 \cdot 3 \mathrm{kDa})$, aldolase $(42.4 \mathrm{kDa})$ and carbonic anhydrase $(30 \mathrm{kDa})$. (b) Western blot of the purified outer membranes. Lanes: 1 , purified OprC; 2 , without added $\mathrm{Cu}^{2+} ; 3,0.1 \mu \mathrm{M} \mathrm{CuSO}_{4} ; 4,1 \mu \mathrm{M}$

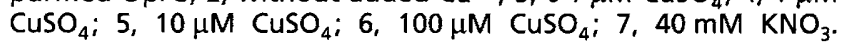
Amounts of protein applied were about $0.1 \mu \mathrm{g}$ in lane $1,5 \mu \mathrm{g}$ in lanes $2-6$, and $1 \mu \mathrm{g}$ in lane 7 . The arrowhead indicates the location of authentic OprC.

putative anr recognition site and because Nos $A$ is induced under anaerobic conditions in $P$. stutzeri, we tested the effect of anaerobiosis on the expression of OprC. Fig. 5
OprC was first identified as one of three porins (OprC, OprD2 and OprE1) in the outer membrane of $P$. aeruginosa that allow the diffusion of small hydrophilic solutes with $M_{\mathrm{r}}$ below 350-400 (Yoshihara \& Nakae, 1989). To confirm this function, we determined the ion conductivity through the OprC channel by incorporating it into planar lipid bilayers. OprC in $1 \mathrm{M} \mathrm{NaCl}$ showed mean conductance of $200 \mathrm{pS}$ and $400-450 \mathrm{pS}$ (Fig. 1). Small and large conductance values were close to those of OprE1 (Obara \& Nakae, 1992) and OprD2 (Ishii \& Nakae, 1993), respectively, of $P$. aeruginosa. Flickering of the channel was often observed at a conductance $200 \mathrm{pS}$ in $1 \mathrm{M} \mathrm{NaCl}$ (Fig. 1c). Therefore, the unit conductance of OprC seemed to be $200 \mathrm{pS}$. Alternatively, it is possible that OprC can take two conformational states that create two different sizes of channel. Two states of channel size 
have been observed in the OMPs LamB of E. coli (Benz et al., 1986) and OprD2 of $P$. aeruginosa (Ishii \& Nakae, 1993)

We cloned the gene encoding $\mathrm{OprC}$ using an anti-OprC antibody. The nucleotide sequence of $\operatorname{oprC}$ showed an ORF of 2169 bp encoding a polypeptide with 723 amino acid residues. A search of the DNA data libraries found similarity between $\mathrm{OprC}$ and several bacterial OMPs. The highest degree of similarity found was with the NosA protein of $P$. stutzeri that is indispensable for the supply of $\mathrm{Cu}^{2+}$ to the periplasmic $\mathrm{N}_{2} \mathrm{O}$ reductase (Mokhele $e t$ al., 1987). NosA-deficient mutants of $P$. stutzeri are unable to grow anaerobically using $\mathrm{N}_{2} \mathrm{O}$ as an electron acceptor. A low concentration of $\mathrm{Cu}^{2+}$ in the culture medium repressed $\mathrm{OprC}$ to a minimum level (Fig. 5). OprC expression was specifically repressed in the presence of $\mathrm{Cu}^{2+}$ (data not shown).

It is likely, therefore, that $\mathrm{OprC}$ binds $\mathrm{Cu}^{2+}$ and conveys the ions to an acceptor protein. We tested the copperbinding affinity of $\mathrm{OprC}$ by equilibrium dialysis. We found high affinity binding of OprC with $\mathrm{Cu}^{2+}$ in vitro with a calculated $K_{\mathrm{d}}$ value of $2.6 \mu \mathrm{M}$. These results suggest the involvement of $\mathrm{OprC}$ in copper utilization. A question arose as to whether $\mathrm{Cu}^{2+}$ affects channel activity. Our preliminary measurement of single channel activity in the presence of $\mathrm{Cu}^{2+}$ showed no significant effect.

A question also arises about how the channel-forming activity and the copper-binding property of $\mathrm{OprC}$ are coordinated in copper utilization. We assume that the size of the OprC, D2 and E1 channels are large enough to accommodate the free passage of $\mathrm{Cu}^{2+}$ across the outer membrane and that $\mathrm{Cu}^{2+}$ readily equilibrates in the periplasm. If so, what would be the role of the specific binding of $\mathrm{Cu}^{2+}$ to $\mathrm{OprC}$ ? It is possible that $\mathrm{N}_{2} \mathrm{O}$ reductase cannot utilize free $\mathrm{Cu}^{2+}$ in the periplasm directly. Instead, $\mathrm{N}_{2} \mathrm{O}$ reductase may require an auxiliary protein which binds $\mathrm{Cu}^{2+}$ and conveys it to the reductase. We speculate that $\mathrm{OprC}$ serves as this auxiliary protein. The presence of such auxiliary binding proteins and their roles are well documented in bacterial chemotaxis and the ATP-dependent active transport system (Adler et al., 1973; Doige \& Ames, 1993). This does not mean that OprC may function as a $\mathrm{Cu}^{2+}$-specific channel. In fact, the mutant carrying a defective $\operatorname{opr} C$ gene, constructed by gene replacement, grew anaerobically in the presence of $20 \mathrm{mM} \mathrm{NaNO}{ }_{2}$ (data not shown). Under these conditions, the copper-requiring redox protein, azurin, binds with free $\mathrm{Cu}^{2+}$ in the periplasm and transfers electrons to nitrite reductase (Zannoni, 1989). This indicates that $\mathrm{Cu}^{2+}$ diffuses across the outer membrane even when $\operatorname{oprC}$ is defective. This result supports the notion that $\mathrm{Cu}^{2+}$ equilibrates across the outer membrane readily and is utilized by azurin, indicating that $\mathrm{OprC}$ is not essential for copper entry into the periplasmic space. We speculate that a physiological role for OprC under anaerobiosis is to convey periplasmic $\mathrm{Cu}^{2+}$ efficiently to $\mathrm{N}_{2} \mathrm{O}$ reductase, otherwise it functions as a channel protein.

The sequence of $\mathrm{OprC}$ exhibits statistically significant similarity with some OMPs involved in iron uptake in a ligand-specific manner. FepA functions through proteinprotein interaction with the inner membrane energy transducer protein, TonB (Skare et al., 1993). It is intriguing to speculate that $\mathrm{OprC}$ conveys $\mathrm{Cu}^{2+}$ by interacting with a putative TonB-like protein in $P$. aeruginosa.

There are well characterized copper resistance operon products in Enterococcus birae (Solioz \& Odermatt, 1995) and Pseudomonas syringae (Cha \& Cooksey, 1991). A protein of $P$. syringae, $\mathrm{CopB}$, is an OMP that is induced in the presence of $\mathrm{Cu}^{2+}$. We could not find any similarity between OprC and these copper-resistant proteins in the similarity search analysis. Furthermore, an OprC-deficient mutant showed no difference in copper susceptibility (data not shown). Therefore, the physiological function of OprC is not related to copper susceptibility. This is accordance with the repression of OprC expression in the presence of $\mathrm{Cu}^{2+}$.

\section{ACKNOWLEDGEMENTS}

We are grateful to T. Yamamoto of Juntendo University for his help in determining the $\mathrm{N}$-terminal amino acid sequence of OprC, S. Miyazawa of Gunma University for his help in use of the FLAT DB e-mail network server and E. Vijgenboom of Leiden University for helpful discussion in sequence analysis. This study was supported financially in part by the Ministry of Education of Japan, Kanagawa Academy of Science and Technology, The Mitsui Life Social Welfare Foundation and Tokai University School of Medicine Research Aid.

\section{REFERENCES}

Adler, J., Hazelbauer, G. L. \& Dahl, M. M. (1973). Chemotaxis toward sugars in Eschericbia coli. J Bacteriol 115, 824-847.

Altschul, S. F., Gish, W., Miller, W., Myers, E. W. \& Lipman, D. J. (1990). Basic local alignment search tool. $J$ Mol Biol 215, 403-410.

Benz, R., Janko, K., Boos, W. \& Läuger, P. (1978). Formation of large, ion-permeable membrane channels by the matrix protein (porin) of Escberichia coli. Biochim Biopbys Acta 511, 305-319.

Benz, R., Schmid, A., Nakae, T. \& Vos-Scheperkeuter, G. H. (1986). Pore formation by LamB of Escherichia coli in lipid bilayer membranes. J Bacteriol 165, 978-986.

Bitter, W., Marugg, J. D., de Weger, L. A., Tommassen, J. \& Weisbeek, P. J. (1991). The ferric-pseudobactin receptor PupA of Pseudomonas putida WCS358: homology to TonB-dependent Eschericbia coli receptors and specificity of the protein. Mol Microbiol 5, 647-655.

Büscher, K. H., Cullmann, W., Dick, W., Wendt, S. \& Opferkuch, W. (1987). Imipenem resistance in Pseudomonas aeruginosa is due to diminished expression of outer membrane proteins. I Infect Dis $\mathbf{1 5 6}$, 681-684.

Cha, J. \& Cooksey, D. A. (1991). Copper resistance in Pseudomonas syringae mediated by periplasmic and outer membrane proteins. Proc Natl Acad Sci US A 88, 8915-8919.

Dean, C. R. \& Poole, K. (1993). Cloning and characterization of the ferric enterobactin receptor gene (PfeA) of Pseudomonas aeruginosa. J Bacteriol 175, 317-324. 
Deretic, V., Konyecsni, W. M., Mohr, C. D., Martin, D. W. \& Hibler, N. S. (1989). Common denominators of promoter control in Pseudomonas and other bacteria. Bio/Technology 7, 1249-1254.

Doige, C. \& Ames, G. F. L. (1993). ATP-dependent transport systems in bacteria and humans: relevance to cystic fibrosis and multidrug resistance. Annu Rev Microbiol 47, 291-319.

Galimand, M., Gamper, M., Zimmermann, A. \& Haas, D. (1991). Positive FNR-like control of anaerobic arginine degradation and nitrate respiration in Pseudomonas aeruginosa. $J$ Bacteriol 173, $1598-1606$.

Heller, K. \& Kadner, R. J. (1985). Nucleotide sequence of the gene for the vitamin B12 receptor protein in the outer membrane of Escbericbia coli. J Bacteriol 161, 904-908.

Hirano, H. \& Watanabe, T. (1990). Microsequencing of proteins electrotransferred onto immobilizing matrices from polyacrylamide gel electrophoresis: application to an insoluble protein. Electrophoresis 11, 573-580.

Hirayama, H. (1976). Spectrophotometric determination of trace amounts of copper based on its catalytic oxidation of $o$-aminophenol. J Chem Soc Jpn 5, 768-772.

Ishii, J. \& Nakae, T. (1993). Lipopolysaccharide promoted opening of the porin channel. FEBS Lett 320, 251-255.

Küpper, H., Sekiya, T., Rosenberg, M., Egan, J. \& Landy, A. (1978). A rho-dependent termination site in the gene coding for tyrosine tRNA su ${ }_{3}$ of Escherichia coli. Nature 272, 423-428.

Laemmli, U. K. (1970). Cleavage of structural proteins during the assembly of the head of bacteriophage T4. Nature 227, 680-685.

Lee, H. S., Hancock, R. E. W. \& Ingraham, J. L. (1989). Properties of a Pseudomonas stutzeri outer membrane channel-forming protein (Nos A) required for production of copper-containing $\mathrm{N}_{2} \mathrm{O}$ reductase. J Bacteriol 171, 2096-2100.

Lee, H. S., Abdelal, A. H. T., Clark, M. A. \& Ingraham, J. L. (1991). Molecular characterization of nos $A$, a Pseudomonas stutzeri gene encoding an outer membrane protein required to make coppercontaining $\mathrm{N}_{2} \mathrm{O}$ reductase. $J$ Bacteriol 173, 5406-5413.

Lipman, D. J. \& Pearson, W. R. (1985). Rapid and sensitive protein similarity searches. Science 227, 1435-1441.

Lundrigan, M. D. \& Kadner, R. J. (1986). Nucleotide sequence of the gene for the ferrienterochelin receptor FepA in Escherichia coli: homology among outer membrane receptors that interact with TonB. J Biol Chem 261, 10797-10801.

Mokhele, K., Tang, Y. J., Clark, M. A. \& Ingraham, J. L. (1987). A Pseudomonas stutzeri outer membrane protein inserts copper into $\mathrm{N}_{2} \mathrm{O}$ reductase. J Bacteriol 169, 5721-5726.

Nau, C. D. \& Konisky, J. (1989). Evolutionary relationship between the TonB-dependent outer membrane transport proteins: nucleotide and amino acid sequences of the Escherichia coli Colicin I receptor gene. J Bacteriol 171, 1041-1047.

Obara, M. \& Nakae, T. (1992). Porin of Pseudomonas aeruginosa forms low conductance ion channel in planar lipid bilayers. Biocbem Biopbys Res Commun 186, 645-651.

Pearson, W. R. \& Lipman, D. J. (1988). Improved tools for biological sequence comparison. Proc Natl Acad Sci USA 85, 2444-2448.

Quinn, J. P., Dudek, E. J., DiVincenzo, C. A., Lucks, D. A.\& Lerner, S. A. (1986). Emergence of resistance to imipenem during therapy for Pseudomonas aeruginosa infections. I Infect Dis 154, 289-294.

Rosenberg, M. \& Court, D. (1979). Regulatory sequences involved in the promotion and termination of RNA transcription. Annu Rev Genet 13, 319-335.
Sambrook, J., Fritsch, E. F. \& Maniatis, T. (1989). Molecular Cloning: a Laboratory Manual, 2nd edn. Cold Spring Harbor, NY: Cold Spring Harbor Laboratory.

Sanger, F., Nicklen, S. \& Coulson, A. R. (1977). DNA sequencing with chain-terminating inhibitors. Proc Natl Acad Sci USA 74, 5463-5467.

Satake, S., Yoshihara, E. \& Nakae, T. (1990). Diffusion of $\beta$-lactam antibiotics through liposome membrane reconstituted from purified porins of the outer membrane of Pseudomonas aeruginosa. Antimicrob Agents Chemotber 34, 685-690.

Sauer, M., Hantke, K. \& Braun, V. (1990). Sequence of the fbuE outer-membrane receptor gene of Eschericbia coli $\mathrm{K} 12$ and properties of mutants. Mol Microbiol 4, 427-437.

Skare, J. T., Ahmer, B. M. M., Seachord, C. L., Darveau, R. P. \& Postle, K. (1993). Energy transduction between membranes. TonB, a cytoplasmic membrane protein, can be chemically cross-linked in vivo to the outer membrane receptor FepA. J Biol Chem 268, 16302-16308.

Solioz, M. \& Odermatt, A. (1995). Copper and silver transport by CopB-ATPase in membrane vesicles of Enterococcus birae. I Biol Cbem 270, 9217-9221.

Toschka, H. Y., Höpfl, P., Ludwig, W., Schleifer, K. H., Ulbrich, N. \& Erdmann, V. A. (1988). Complete nucleotide sequence of a $16 \mathrm{~S}$ ribosomal RNA gene from Pseudomonas aeruginosa. Nucleic Acids Res 16, 2348.

Trias, J. \& Nikaido, H. (1990). Outer membrane protein D2 catalyzes facilitated diffusion of carbapenems and penems through the outer membrane of Pseudomonas aeruginosa. Antimicrob Agents Chemother 34, 52-57.

Yamano, Y., Nishikawa, T. \& Komatsu, Y. (1993). Cloning and nucleotide sequence of anaerobically induced porin protein E1 (OprE) of Pseudomonas aeruginosa PAO1. Mol Microbiol 8, 993-1004. Yoneyama, H. \& Nakae, T. (1986). A small diffusion pore in the outer membrane of Pseudomonas aeruginosa. Eur J Biochem 157, 33-38. Yoneyama, H. \& Nakae, T. (1991). Cloning of the protein D2 gene of Pseudomonas aeruginosa and its functional expression in the imipenem-resistant host. FEBS Lett 283, 177-179.

Yoneyama, H., Akatsuka, A. \& Nakae, T. (1986). The outer membrane of Pseudomonas aeruginosa is a barrier against the penetration of disaccharides. Biocbem Biopbys Res Commun 134, $106-112$.

Yoneyama, H., Yoshihara, E. \& Nakae, T. (1992). Nucleotide sequence of the protein D2 gene of Pseudomonas aeruginosa. Antimicrob Agents Chemother 36, 1791-1793.

Yoshihara, E. \& Nakae, T. (1989). Identification of porins in the outer membrane of Pseudomonas aeruginosa that form small diffusion pores. J Biol Chem 264, 6297-6301.

Yoshihara, E. \& Nakae, T. (1992). Separation of gate- and channelforming domains in the pore-forming protein of the outer membrane of Pseudomonas aeruginosa. FEBS Lett 306, 5-8.

Yoshihara, E., Yoneyama, H. \& Nakae, T. (1991). In vitro assembly of the functional porin trimer from dissociated monomers in Pseudomonas aeruginosa. J Biol Chem 266, 952-957.

Zannoni, D. (1989). The respiratory chains of pathogenic pseudomonads. Biochim Biopbys Acta 975, 299-316.

Received 6 November 1995; revised 14 March 1996; accepted 20 March 1996. 